\title{
BMJ Open Quality Medic One Pediatric (MOPed) cards: standardising paramedic paediatric resuscitation
}

Brandon Woods, ${ }^{\oplus 1}$ Benjamin Lang, ${ }^{2,3}$ Carolyn Blayney, ${ }^{3}$ Lila O'Mahony, ${ }^{1}$
Amanda Vander Tuig, ${ }^{4}$ Tom Rea, ${ }^{5}$ David Carlbom, ${ }^{6}$ Michael Sayre, ${ }^{5}$ Mary King

\section{ABSTRACT}

Objective Paediatric resuscitation is highly stressful, technically challenging and infrequently performed by paramedics. Length-based equipment selection, weightbased medication dosing and less familiar paediatric clinical scenarios create high cognitive load. Our project aimed to decrease cognitive load and increase paramedic comfort by providing standardised paediatric resuscitation cards across an entire Emergency Medical Services (EMS) system.

Methods After 2 years of collaboration between EMS and regional paediatric subspecialists, we created and implemented a novel set of length-based, colour-coded cards: Medic One Pediatric (MOPed) cards. MOPed cards standardise the approach to paediatric scenarios, such as rapid sequence intubation (RSI), seizure management and cardiac arrest. We standardised drug concentrations across all five EMS agencies to allow for volume-based dosing, removing medication calculations, simplifying the process of medication administration and potentially decreasing both calculation error and time to intervention. We consolidated medications on MOPed cards to the 12 most commonly used in Paediatric Advanced Life Support scenarios. We surveyed 240 EMS personnel before and after implementation to determine use and effect on paramedic comfort.

Results After 12 months of implementation, $97 \%$ of respondents reported using the new cards as their primary reference, and $94 \%$ reported improved speed and accuracy of medication administration. Specifically, $\mathrm{RSI}$ medication administration received the greatest improvement in comfort $(p=0.001)$. Additionally, paramedics increased the use of MOPed cards when selecting endotracheal tubes: $45 \%$ of the respondents had done so by 6 months, and $60 \%$ had done so after 12 months of implementation $(p=0.01)$.

Conclusions MOPed cards were well adopted across a large EMS system, with improvement in paramedic comfort in managing some paediatric resuscitation scenarios.

\section{INTRODUCTION}

\section{Problem description}

In the prehospital emergency setting, paediatric resuscitation is a high-stress, low-frequency and technically challenging event. Clinical scenarios often require paediatric resuscitation and include respiratory distress, respiratory failure, cardiac arrest, shock (hypovolemic, septic, neurogenic or obstructive) and status epilepticus. Compared with adult cardiac arrest resuscitation, paediatric resuscitation has additional complexity related to medication dosing, sizing and selection of equipment, and an extra emotional component, leading to heightened anxiety for providers. These differences create a high cognitive load for paramedics unique to paediatric resuscitation. ${ }^{1}$ A high cognitive load may contribute to human error and the potential for poor patient outcomes as evidenced by simulation studies. ${ }^{2}$ A study of paediatric resuscitation observed an overall medication error prevalence of $>30 \%{ }^{3}$ and an epinephrine specific error prevalence of $>60 \%{ }^{3}$

As part of our quality improvement (QI) programme at our large Level 1 Trauma Centre, we implemented both in-patient and prehospital initiatives to achieve consistency in medication administration. We launched an in-hospital and regional effort to colourcode every paediatric patient with every admission and use colour coding for dosing in the field and throughout patient hospitalisation. We used the plan, do, study, act (PDSA) model of QI. Initially, colour-coded stickers (see figure 1) were placed on highrisk medications, such as epinephrine, to improve speed and accuracy of administration in-hospital (our initial objective-the 'do' of PDSA). Exploring potential prehospital use, we performed a high-fidelity simulation scenario to assess time to epinephrine for a paediatric patient with cardiac arrest using colour-coded epinephrine dose stickers versus the paramedics' typical method (simulation performed by author MA King, 19-22 November 2013, at the King County Medic One Training Conference) ('study' in the PDSA cycle). Paramedics were given the opportunity to use their traditional resources to determine epinephrine dosing, including 


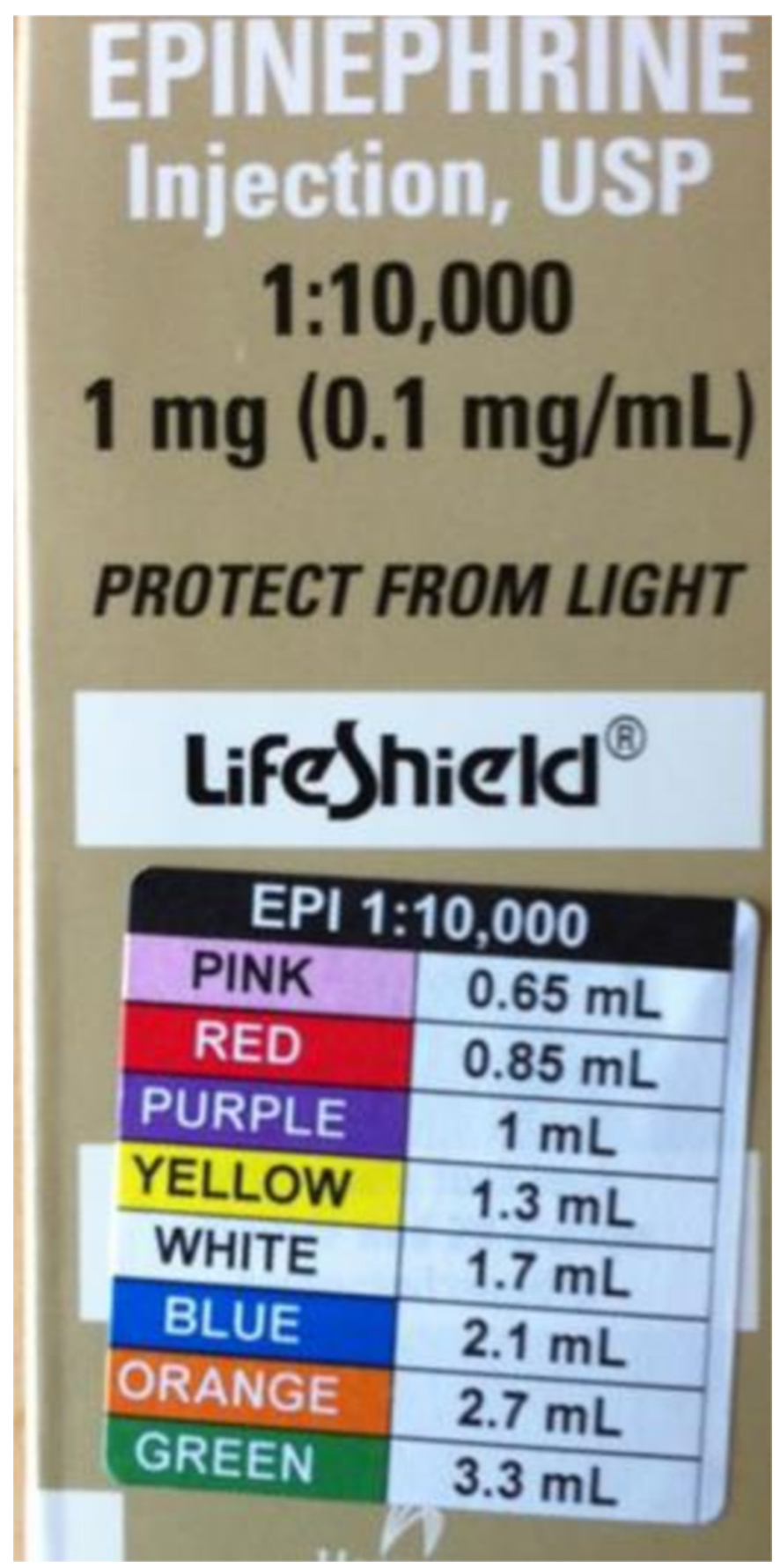

Figure 1 Colour-coded labels on high-risk medications.

the Broselow tape, iPhone ${ }^{\odot}$ applications, a King County emergency medical services (EMS) handbook, personal memory and recently implemented colour-coded stickers. In this scenario, we found a wide variation in practice for the determination of epinephrine dosing.

In addition, the simulation scenario included paediatric endotracheal intubation, which also showed variation in practice among medication selection and dosage, depending on the resuscitation aid used and the lack of standardised algorithm. Despite our high successful rate of intubation, ${ }^{4}$ we acknowledged that further improvements could be made in medication selection and administration. Following this simulation exercise, which demonstrated a large variation in practice, paramedic leadership directly approached our team to create simple colour-coded cards that could precalculate medication doses and standardise paramedic paediatric resuscitation ('act' in the PDSA cycle).

\section{Available knowledge}

Cognitive load is a critical element of the paediatric resuscitation process. ${ }^{1}$ Cognitive load is defined as the amount of mental work required to recall and act on content knowledge and to make decisions under time pressure. ${ }^{56}$ Cognitive load depends on the degree of uncertainty and is greater when the task is less familiar and more complex. ${ }^{1}$ Those with less experience also have higher cognitive load in prior studies. ${ }^{7}$ Cognitive load is also a significant factor in other aspects of medicine, such as patient handoffs and education. ${ }^{8}$ In the setting of paediatric resuscitation, size-related variables introduce the need for non-automatic activities and decisions, thereby increasing the cognitive load. The size-related variables are unique to the resuscitation of children. These nonautomatic, size-related decisions can be relegated to an automatic level using resuscitation aids. ${ }^{1}$

Increased cognitive loading can translate to increased risk of error. ${ }^{1}$ Increasing cognitive load associated with paediatric $^{9}$ and neonatal ${ }^{5}$ resuscitations leads to serious safety events, often composed of medication errors or delay in medication administration. For example, longer time to epinephrine has been associated with increased mortality in the setting of cardiac arrest in both the inpatient $^{1011}$ and outpatient environments. ${ }^{12} 13$

Conversely, there have been several advancements that have mitigated cognitive load, but none have combined these efforts into a resuscitation reference that included EMS input and was well adopted across an entire EMS system. The Broselow tape method has been shown to be accurate in the estimation of weight for the calculation of medication doses. ${ }^{14}{ }^{15}$ Furthermore, using colourcoded administration of medications ${ }^{16-18}$ across an entire EMS system is also beneficial. ${ }^{19}$ Resuscitation aids, such as reference cards, have also improved resuscitation. ${ }^{20} 21$ In a recent national survey of paramedics with over 1000 responses, paramedics cited EMS-specific Broselow-Luten tape as helpful in reducing paediatric drug dosing errors $(89 \%)$, as well as drug dosing cards in millilitres $(83.0 \%){ }^{3}$

\section{Rationale}

We wanted to combine previous efforts to decrease cognitive load in a novel set of length-based cards and to implement them across an entire EMS system. The concept of cognitive load theory has been applied to the prehospital medical community. ${ }^{22}$ There have been efforts at reducing cognitive load in other situations using standardisation and simplification. Our goal was to reduce paramedic cognitive load via creation and implementation of a standardised system that decreases the number of decisions and the amount of uncertainty by (1) reducing the number of drug choices, (2) simplifying 
and standardising care algorithms, and (3) precalculating drug volumes.

\section{Specific aim}

Our primary aim was to collaboratively develop and implement novel paediatric resuscitation reference cards for paramedics that were well-adopted across all Medic One Paramedics in King County, Washington. We aimed to make the MOPed cards simple in readability and application, portable and transferrable to inpatient practices, volume based in dosing reference and consistent with Paediatric Advanced Life Support (PALS) recommendations and well-adopted by the end users: paramedics. Our secondary aim was to evaluate the effect of MOPed card implementation and use on paramedic comfort during paediatric resuscitation.

\section{METHODS}

\section{Context}

Paramedics in King County are specifically trained with an emphasis on critical care patient management and skills. The EMS system is a tiered response that enables a relatively modest number of paramedics $(n=240)$ to serve a relatively large population: 2.1 million persons with 360000 children less than 12 years. ${ }^{23}$ Paramedics have stringent requirements for ongoing education, including PALS every 2 years. On average, individual paramedics treat approximately eight cardiac arrests and perform 12 endotracheal intubations annually (adult and paediatric). Given this training and experience, paramedics achieve a high level of critical care success. In a review of the King County paramedic experience, ${ }^{5}$ we found paramedics perform paediatric rapid sequence intubation (RSI) infrequently (299 cases over 6.3 years, excluding Seattle) but have a $97 \%$ overall success rate. ${ }^{5}$ Yet, paramedics acknowledge that management of the critically ill paediatric patient is one of the most challenging scenarios for which delays and even errors are possible.

\section{Intervention}

We convened a group of regional experts to review current paediatric reference materials for paramedic care with the goal of consolidating information into an efficient and user-friendly resource. The group included paediatric and adult critical care physicians and nurses, paediatric emergency medicine physicians, adult emergency medicine physicians and pharmacists. As part of the process, the paediatric working group met with paramedics and EMS medical directors to gain consensus on the form and format, as well as to determine specifics about medication and equipment availability and prehospital treatment guidelines.

This working group developed a prototype resource that consisted of colour-coded cards with medication doses chosen by cross-referencing our institution's and Broselow code sheets, PALS recommendations and vetting medication choices with the EMS and paediatric subspecialist group (see figure 2). The MOPed cards were

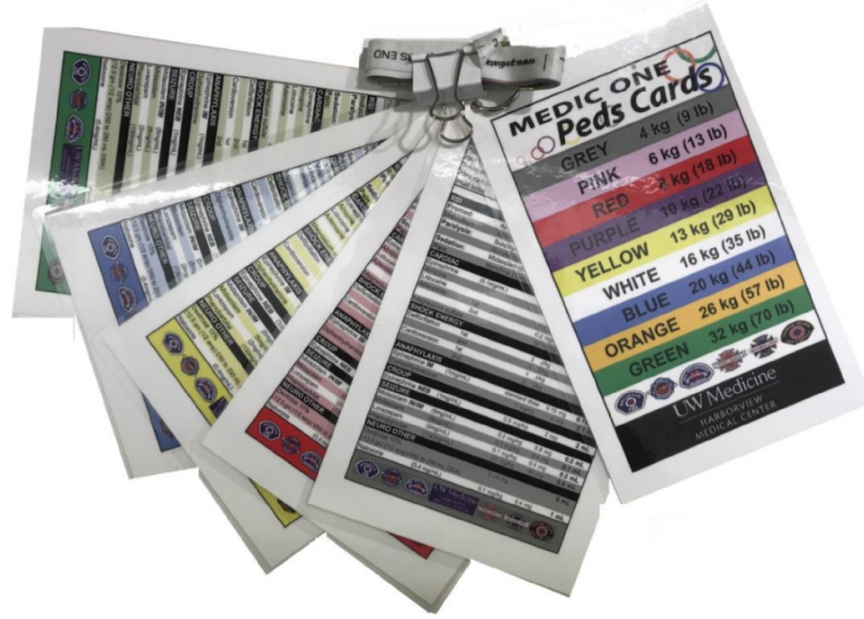

Figure 2 Medic One Pediatric (MOPed) cards.

designed as an airway, breathing, circulation, disability resuscitation tool based on PALS for critically ill children in King County. We included medications to support the care for paediatric patients in the scenarios that presented in King County, including airway/breathing (paediatric RSI, anaphylaxis and croup), cardiac (cardiac arrest and arrythmia) and disability (seizure, hypoglycaemia and toxicology). These decisions were based on review of a recent analysis of paediatric ALS runs, ${ }^{5}$ as well as EMS medical director input.

Each medication was given in weight-based dose (mg/ $\mathrm{kg})$, absolute milligram dose and volume $(\mathrm{mL})$ dose with volume dose being bolded. To enable volume dosing, we collaborated with EMS leadership to ensure that all agencies were using the same formulary and drug concentrations. The MOPed card paediatric subspecialty team visited EMS paramedic medical director meetings four times over the course of approximately 24 months (20142016) (see figure 3) to iteratively present card drafts and recommendations and to obtain paramedic and medical director input to enable card acceptance across all five paramedic agencies while ensuring their consistency with PALS guidelines and regional paediatric subspecialty recommendations. The initial meeting was a presentation about a potential MOPed card project by the MOPed team. Input was obtained and collaboratively agreed to over the course of the next two meetings on four distinct objectives: (1) whether we would be able to standardise drug formularies; (2) whether directors would support a standardised approach to paediatric RSI and paediatric seizure; (3) which medications that used to be a standard part of paediatric cardiac arrest resuscitation were no longer necessary, such as calcium and bicarbonate; and (4) which content areas providers would want to learn more about (seizure, medications and route). After these decisions were made as a group, additional input was obtained by the MOPed team from regional paediatric subspecialists, including paediatric anaesthesia, paediatric critical care and paediatric neurology. A new version of MOPed was created that included precalculation of 


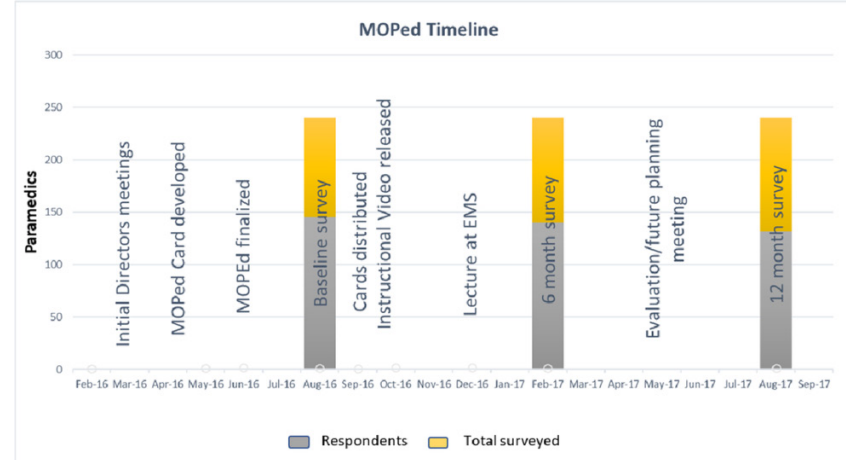

Figure 3 MOPed timeline. EMS, emergency medical services; MOPed, Medic One Pediatric.

medications. At the third medical director meeting, this version was reviewed and agreed to with continued small changes, including formatting. Lastly, at the fourth meeting, the MOPed cards were delivered to the various agency medical directors, and education was provided, including a request for a video educational format for medics at the time of rollout. A list of drug concentrations was also included as an additional safety measure. Prior to distribution, an instructional video was created and distributed that described card use for the paremedic.

\section{Study of intervention}

We distributed the MOPed cards across all five paramedic agencies in September 2016. To evaluate the effect of the cards on paramedic comfort with paediatric resuscitation, we distributed surveys preintervention, after 6 months and after 12 months of implementation. Surveys were distributed electronically to all 240 paramedics in King County, and results were collected in an anonymous fashion. To both augment MOPed card awareness and provide paramedic paediatric education (MOPed case review, education and feedback), the MOPed team gave a MOPed card training lecture at the University of Washington Harborview Medical Center Paramedic Training Programme on 6 December 2016 (4 months after MOPed implementation).

\section{Measures}

As mentioned, we distributed surveys preintervention, after 6 months and after 12 months of implementation. The survey had two main analytical goals: (1) paramedic current use of resuscitation aids, including the MOPed cards, and (2) paramedic comfort in responding to critical care paediatric scenarios that were part of the paramedic scope of practice. Comfort was measured using the Likert scale. As a control, we asked a comfort question regarding adult cardiac arrest. Use of the cards was categorised according to various clinical scenarios and overall use.

\section{Analysis}

We primarily used descriptive statistics to evaluate use and comfort. Likert-type items were coded with the following anchors: $1=$ strongly disagree, $2=$ disagree, $3=$ neither agree nor disagree, $4=$ agree, and $5=$ strongly agree. An aggregate score was calculated by summing the six questions given at each observation. The distributions for each question and the aggregate score were analysed for normality using skewness and kurtosis statistics. Wilcoxon ranked-sum tests were used to compare non-normal distributions in a within-subjects fashion. Repeated-measures t-tests were used to test for significant effects across time for normally distributed observations. Statistical significance was assumed with a Bonferroni-adjusted alpha value of 0.007 (0.05 divided by the seven hypotheses tested) to account for increased experiment-wise error rates. All analyses were conducted using SPSS V.21.

\section{RESULTS}

All 240 paramedics within King County were surveyed electronically via email request of their paramedic leadership. Our preimplementation survey distributed in August 2016 received 145 responses, representing a 60\% response rate. We received 140 responses (58\% response rate) at 6 months and 132 responses (55\% response rate) at 12 months (table 1 ).

Demographic responses to the survey demonstrated a wide variety of paramedic experience: $10 \%$ respondents had $0-3$ years, $21 \%$ had $3-10$ years, $21 \%$ had $10-15$ years and $47 \%$ had greater than 15 years' paramedic experience. Experience was grossly unchanged between survey time points.

Medic survey demonstrated a great increase in the use of the MOPed cards throughout the project. Prior to implementation, our paramedics reported using eight different resuscitation references. The Broselow tape was the most commonly used, with $75 \%$ reporting its use (table 2). Hard copy formularies were the second most common aid. After 6 months, $85 \%$ reported using MOPed cards as a reference, with $95 \%$ reporting using it as their primary reference for paediatric resuscitation and equipment selection. This improved to $88 \%$ and $97 \%$, respectively, at the 12-month survey. After 6 months of implementation,

Table 1 Survey response rates

\begin{tabular}{llll}
\hline & Preimplementation & Mid-implementation & Postimplementation \\
\hline Survey time point & Baseline & $\mathbf{6}$ months & $\mathbf{1 2}$ months \\
Paramedics surveyed & 240 & 240 & 240 \\
Responses & 145 & 140 & 132 \\
Response rate & $60 \%$ & $58 \%$ & $55 \%$ \\
\hline
\end{tabular}


Table 2 Survey responses for resuscitation

\begin{tabular}{|c|c|c|c|c|}
\hline & Pre (\%) & Mid (\%) & Post (\%) & P (mid to post) \\
\hline \multicolumn{5}{|l|}{$\begin{array}{l}\text { What reference do you use for obtaining medication doses and } \\
\text { equipment size for children? }\end{array}$} \\
\hline MOPed cards & - & 85 & 88 & - \\
\hline Device application & 9 & 1 & 1 & - \\
\hline Other & 14 & 7 & 8 & - \\
\hline $\begin{array}{l}\text { I now use the MOPed cards as my primary reference for paediatric } \\
\text { resuscitation and equipment. }\end{array}$ & - & 95 & 97 & 0.75 \\
\hline $\begin{array}{l}\text { I felt more comfortable with the MOPed compared with my previous } \\
\text { method. }\end{array}$ & - & 85 & 89 & 0.55 \\
\hline $\begin{array}{l}\text { I feel the MOPed cards improved my speed and the accuracy of } \\
\text { endotracheal tube selection. }\end{array}$ & - & 73 & 86 & 0.11 \\
\hline $\begin{array}{l}\text { I used the MOPed cards reference in order to select an appropriately } \\
\text { sized endotracheal tube for a paediatric patient ( }<12 \text { years old). }\end{array}$ & - & 45 & 60 & 0.01 \\
\hline $\begin{array}{l}\text { I used the MOPed cards to select appropriate weight-based dosages } \\
\text { of rapid sequence intubation medications. }\end{array}$ & - & 53 & 61 & 0.14 \\
\hline $\begin{array}{l}\text { I used the MOPed cards to select appropriate weight-based dosages } \\
\text { of antiepileptic medications. }\end{array}$ & - & 65 & 74 & 0.11 \\
\hline $\begin{array}{l}\text { I used the MOPed cards to select appropriate weight-based dosages } \\
\text { of epinephrine for cardiac arrest. }\end{array}$ & - & 42 & 52 & 0.13 \\
\hline
\end{tabular}

MOPed, Medic One Pediatric.

$45 \%$ responded that they had used MOPed cards to select an endotracheal tube, which increased to $60 \%$ respondents after 12 months $(p=0.01)$. For selecting antiepileptic medication doses, $65 \%$ of respondents reported use of MOPEed cards at 6 months and $74 \%$ reported use at 12 months $(\mathrm{p}=0.11)$. MOPed cards were also used to select appropriate weight-based doses of epinephrine for cardiac arrest, with $42 \%$ respondents reporting use at 6 months, and 52\% ( $\mathrm{p}=0.13$ ) at 12 months.

When queried regarding overall comfort with the cards compared with their previous method, $85 \%$ responded that they felt more comfortable after 6 months of implementation and $89 \%$ felt more comfortable at 12 months. Ninety-one per cent felt that MOPed cards improved their speed and accuracy of medication administration after 6 months, similar to $94 \%$ at the 12 -month survey $(\mathrm{p}=0.62)$. We also observed improvement with perceived speed and accuracy of endotracheal tube size selection, with $73 \%$ reporting improvement at 6 months and 86\% reporting improvement after 12 months $(\mathrm{p}=0.11)$.

We asked comfort questions specific to scenarios (table 3). For our control question, 'I feel comfortable managing an adult with cardiac arrest including the post-arrest period', $89 \%$ responded they strongly agreed preintervention and $87 \%$ responded 12 months postintervention, suggesting consistency among survey respondents. When asked the same cardiac arrest scenario with a child, we saw a slight improvement. Eighty percent were either in strong agreement or agreement preintervention, and $85 \%$ after 12 months of intervention $(\mathrm{p}=0.301)$. We asked paramedics if they felt comfortable identifying and giving weight-based dosages of RSI medications, with a repeated paired t-test value of $\mathrm{p}=0.001$ (see figure 4).

\section{DISCUSSION}

\section{Summary}

Through multidisciplinary collaboration, we were able to develop and implement novel paediatric critical care cards across a large metropolitan EMS system, with 97\% reporting MOPed cards as their primary reference 12 months following implementation. We were able to improve comfort in some clinical scenarios, specifically RSI medication administration, but not in other critical care scenarios, such as seizure management and cardiac arrest resuscitation. We attribute the improvement to the simplicity of our cards, with a reduction in the number of medications and volume dosing. Paramedic comfort is not equivalent to actual outcomes of paediatric resuscitation. Thus, it will be helpful to explore relationships 
between the actual performance measures of these paediatric resuscitations to the paramedic's overall perception.

The MOPed cards were successful with the collaboration from paramedics, EMS directors, medical control physicians and paediatric subspecialists. The paramedics themselves were a driving force in MOPed card initiation, development and roll-out, and we believe this engagement was a fundamental key to EMS leadership buy-in and broad paramedic support. Working at a mixed adult and paediatric level i trauma centre, we were uniquely positioned to have close relationships between EMS and paediatric providers. These connections enabled a natural QI discussion and follow-up, including EMS education and simulation. This project demonstrates the importance of cultivating these interdisciplinary relationships. Standardising the drug formulary and drug concentrations across paramedic agencies in King County was also key to the development of the MOPed cards.

In addition to paramedics' standard use of MOPed cards, we also successfully changed the inpatient and outpatient medication processes at our level 1 paediatric trauma centre using the same colour-coding regimen from initial field resuscitation through the entire hospital length of stay. This approach created a streamlined process that inherently built safety into medication administration and lent itself well to paediatric disaster preparedness.

I feel comfortable identifying and giving weight-based dosages of rapid sequence intubation medications

PRE

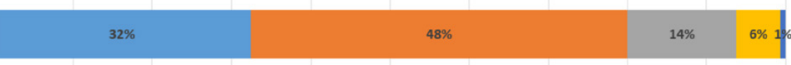

POST

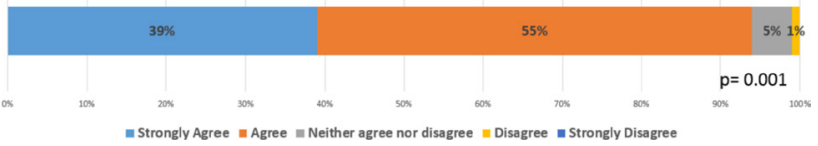

Figure 4 Rapid sequence intubation improvement.
Given the framework of collaboration and the PDSA model of improvement, we envision sustaining improvement with paediatric resuscitation in King County with continued modifications of the MOPed cards, as well as development of a MOPed card specific to inpatient use. We collected many comments in the surveys that can be incorporated into future iterations of the MOPed cards. We were able to perform subsequent paramedic education in between the survey intervals. Additionally, we provided direct email access to the MOPed team at all times during this project. During a critical medication shortage of morphine, our team was able to quickly convene and create an emergency fentanyl MOPed card and distribute it electronically to all paramedic ambulances.

\section{Limitations}

This study is limited by issues involving survey research, including responder bias. Respondents were given as much time as needed to complete the survey, but there may have been inaccurate reporting due to recall bias of events that occurred up to 6 months previously. To protect the anonymity of the respondents, no identifying information was collected.

Limited data exist on implementing the resuscitation aids on scales similar to those in our project. MOPed cards are designed to be primarily used within the prehospital setting. They are not designed for a paediatric emergency department as they are designed to contain a limited medication formulary commonly used in EMS agencies. If a hospital or clinic sees children infrequently, the simplicity of these cards and the subsequent decreased cognitive load may outweigh the potential weakness of having limited medication formulary.

There is the potential for dosing error should a drug concentration be used that is different from the concentration indicated on the cards. Thus, it is of critical importance that system managers first verify they have the 
correct drug concentrations before deploying MOPed cards. Efforts were made throughout to ensure singular concentrations across the EMS system.

Lastly, this study does not measure MOPed card efficacy on paediatric resuscitation itself as the goal of this investigation was to gauge acceptance and use of the new paediatric resource. It lacks the quantitative data on timing and accuracy of equipment selection and medication administration. These data are currently being collected and will be evaluated to determine MOPed card efficacy as compared with prior practice in a future study.

\section{CONCLUSIONS}

Our project highlights the importance of collaboration to bring about change. The new paediatric resuscitation cards were well adopted in a collaborative manner across a large EMS system, with improvement in paramedic comfort in managing several paediatric resuscitation scenarios. For further evaluation, quantitative data on timing and accuracy of equipment selection and medication administration, as well as patient outcomes, should be evaluated to further determine efficacy as compared with prior practice.

Contributors BW conceptualised and designed the study, participated in data collection and data analysis, and drafted the initial manuscript; BL participated in data collection and analysis; $\mathrm{CB}$ and AV conceptualised and developed the MOPed cards; LO'M conceptualised and designed the study; DC, TR and MS designed and conceptualised the study and provided manuscript revisions; MK conceptualised and designed the study, participated in data analysis and assisted in the initial manuscript; and all authors critically reviewed and revised the manuscript and approved the final manuscript as submitted.

Funding All phases of this study were supported by a grant from the Central Region Emergency Medical Service/Trauma Council.

Competing interests None.

Patient consent for publication Not required.

Ethics approval Paramedic surveys were performed in bulk, deidentified fashion, and the study was institutional review board-approved by the Seattle Children's Institutional Review Board.

Provenance and peer review Not commissioned; externally peer reviewed.

Open access This is an open access article distributed in accordance with the Creative Commons Attribution Non Commercial (CC BY-NC 4.0) license, which permits others to distribute, remix, adapt, build upon this work non-commercially, and license their derivative works on different terms, provided the original work is properly cited, appropriate credit is given, any changes made indicated, and the use is non-commercial. See: http://creativecommons.org/licenses/by-nc/4.0/.

\section{REFERENCES}

1. Luten R, Wears RL, Broselow J, et al. Managing the unique sizerelated issues of pediatric resuscitation: reducing cognitive load with resuscitation AIDS. Acad Emerg Med 2002;9:840-7.
2. Lammers RL, Byrwa MJ, Fales WD, et al. Simulation-based assessment of paramedic pediatric resuscitation skills. Prehosp Emerg Care 2009;13:345-56.

3. Hoyle JD, Davis AT, Putman KK, et al. Medication dosing errors in pediatric patients treated by emergency medical services. Prehosp Emerg Care 2012;16:59-66.

4. Prekker ME, Delgado F, Shin J, et al. Pediatric intubation by Paramedics in a large emergency medical services system: process, challenges, and outcomes. Ann Emerg Med 2016;67:20-9. 2016.

5. Yamada NK, Yaeger KA, Halamek LP. Analysis and classification of errors made by teams during neonatal resuscitation. Resuscitation 2015;96:109-13.

6. Sweller J. Cognitive load during problem solving: effects on learning. Cogn Sci 1988;12:257-85.

7. Szulewski A, Gegenfurtner A, Howes DW, et al. Measuring physician cognitive load: validity evidence for a physiologic and a psychometric tool. Adv in Health Sci Educ 2017;22:951-68.

8. Young JQ, Wachter RM, ten Cate O, et al. Advancing the next generation of handover research and practice with cognitive load theory. BMJ Qual Saf 2016;25:66-70.

9. Meckler G, Hansen M, Lambert W, et al. Out-Of-Hospital pediatric patient safety events: results of the CsI chart review. Prehosp Emerg Care 2018;22:290-9.

10. Andersen LW, Berg KM, Saindon BZ, et al. Time to epinephrine and survival after pediatric in-hospital cardiac arrest. JAMA 2015;314:802-10.

11. Doherty C, Mc Donnell C. Tenfold medication errors: 5 years' experience at a university-affiliated pediatric hospital. Pediatrics 2012;129:916-24.

12. Fukuda $\mathrm{T}$, Kondo $\mathrm{Y}$, Hayashida $\mathrm{K}$, et al. Time to epinephrine and survival after paediatric out-of-hospital cardiac arrest. Eur Heart $J$ Cardiovasc Pharmacother 2018;4:144-51.

13. Goto Y, Maeda T, Goto Y. Effects of prehospital epinephrine during out-of-hospital cardiac arrest with initial non-shockable rhythm: an observational cohort study. Crit Care 2013;17.

14. Bourdeau S, Copeland J, Milne WK. Accuracy of the Broselow tape in estimating the weight of first nations children. Can J Rural Med 2011;16:121-5.

15. Lubitz DS, Seidel JS, Chameides L, et al. A rapid method for estimating weight and resuscitation drug dosages from length in the pediatric age group. Ann Emerg Med 1988;17:576-81.

16. Stevens AD, Hernandez C, Jones S, et al. Color-Coded prefilled medication syringes decrease time to delivery and dosing errors in simulated prehospital pediatric resuscitations: a randomized crossover trial. Resuscitation 2015;96:85-91.

17. DeBoer S, Seaver M, Broselow J. Color coding to reduce errors: the Broselow-Luten system streamlines pediatric emergency treatment. Am J Nurs 2005;105:68-71.

18. Shah AN, Frush K, Luo X, et al. Effect of an intervention standardization system on pediatric dosing and equipment size determination: a crossover trial involving simulated resuscitation events. Arch Pediatr Adolesc Med 2003;157:229-36.

19. Kaji AH, Gausche-Hill M, Conrad H, et al. Emergency medical services system changes reduce pediatric epinephrine dosing errors in the prehospital setting. Pediatrics 2006;118:1493-500.

20. Luten R. Error and time delay in pediatric trauma resuscitation: addressing the problem with color-coded resuscitation AIDS. Surg Clin North Am 2002;82:303-14.

21. Bernius M, Thibodeau B, Jones A, et al. Prevention of pediatric drug calculation errors by prehospital care providers. Prehosp Emerg Care 2008:12:486-94.

22. Lauria MJ, Bronson MR, Lanter PL, et al. The 5 T's: applying cognitive science to improve prehospital medical education. Air Med J 2017;36:198-202.

23. U.S. Census Bureau. 2012-2016 American community survey 5-year estimates. Children characteristics. Available: https://factfinder. census.gov/faces/nav/jsf/pages/community_facts.xhtml?src=bkmk [Accessed 19 Dec 2017]. 